NOUVELLES DONNÉES

SUR LES OSTRACODES D'EAU DOUCE DE ROUMANIE : CORDOCYTHERE PHREATICOLA n. g. n. sp., EUCYPRIS PETKOVSKII n. sp., LIMNOCYTHERINI ET METACYPRINI NOUVELLES TRIBUS DE LA SOUS-FAMILLE LIMNOCYTHERINAE SARS, 1925

\author{
par Dan L. Danielopor.
}

Les Ostracodes d'eau douce ont été peu étudiés en Roumanie, bien que ce groupe soit représenté en Europe par plus de 200 espèces. Grâce aux recherches de Daday [1900], Masi [1906], KLIE [1943], Puscariu [1951], Beldescu [1961 a, 1961 b], Danielopol. et VeSPREMEANU [1964], on connaissait jusqu'à présent en Roumanie 43 espèces.

Dans ce qui suit sont présentées les descriptions de Cordocythere phreaticola n. g. n. sp. et d'Eucypris petkovskii n. sp., ainsi que des remarques systématiques, écologiques et zoogéographiques concernant 13 espèces citées pour la première fois dans la faune de la Roumanie.

J'adresse mes vifs remerciements aux Prof. Dr I. D. Pinto (Escola de Geologia, Porto-Alegre), Dr A. Capart (Institut Royal des Sciences Naturelles de Belgique) et Dr F. Trieber (Senckenberg Museum), qui ont eu l'amabilité de m'envoyer des exemplaires de Cytheridella sp., Elpidium sp., Gomphocythere angusta et Metacypris cordata, absolument nécessaires à mes études comparatives.

Je tiens à remercier aussi le Dr W. Tressien (U. S. Hydrographic Office) pour les précieux renseignements concernant les espèces actuelles du genre Metacypris, ainsi qu'aux Dr J. P. HarDing (British Museum), Dr L. Cahen (Musée Royal de l’Afrique Centrale, Dr D. R. Rome (Université de Louvain), Dr 'T. Petrovski (Musée d'Histoire Naturelle de Skopje), Dr Ann Chapmann (Université de Glasgow), qui ont eu l'obligeance de me procurer des photocopies concernant quelques articles que je ne possédais pas. 


\section{DONNÉES SYSTÉMATIQUES}

\section{I. - FAMILle CYPRIDIDAE W. Baird, 1845.}

A. - Sous-famille ILYOCYPRINAE Kaufmann, 1900.

Ilyocypris decipiens Masi, 1906.

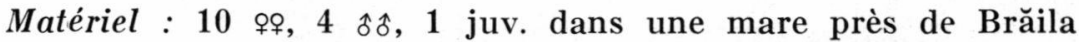
(Reg. Galați), 18.VII.1964.

Parmi les 15 exemplaires étudiés, j'en ai trouvé un dont l'avantdernier article de la troisième patte était muni sur le bord interne de trois poils au lieu de deux (fig. 1 A).

G. W. Müller [1912] et H. Gauthier [1938] ont signalé chez Ilyocypris bradyi SARS des aberrations semblables.

Enfin tous les exemplaires femelles que j'ai disséqués possédaient le palpe de la première patte biarticulé et non triarticulé comme le figure Petkovski [1957].

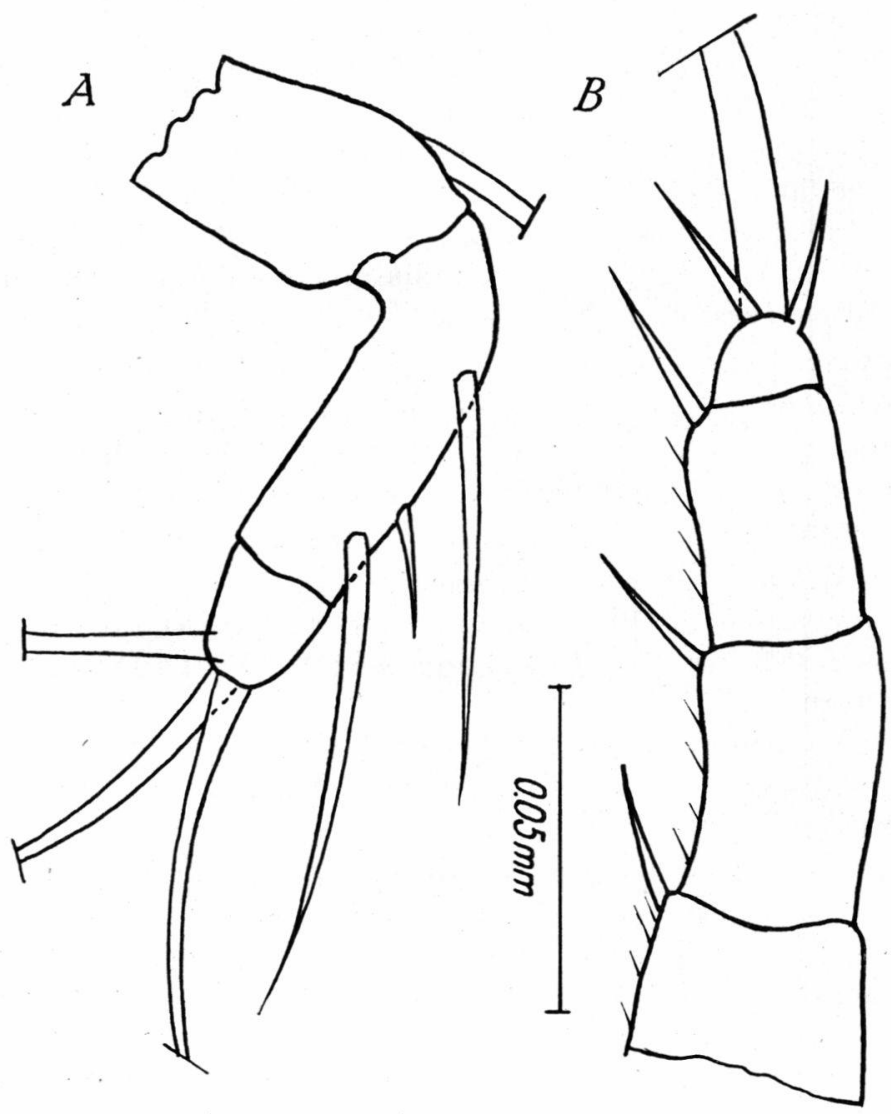

Frg. 1. - Ilyocypris decipiens Ması o. A : $3^{\mathrm{e}}$ patte (détail); $\mathrm{B}: 2^{\mathrm{e}}$ patte (détail). 
B. - Sous-famille CYPRIDINAE Baird, 1845.

\section{1) Tribu Eucypridini.}

\section{Eucypris petkovskii n. sp. ${ }^{1}$.}

\section{Matériel.}

Nombreux exemplaires femelles recueillis dans une mare près de Brăila (à proximité de la localité Vărsătura), 30.X.1964.

\section{Diagnose.}

Une Eucypris de forte taille (2,4 $\mathrm{mm}$ en moyenne), avec des valves asymétriques, la gauche étant plus grande que la droite. Le bord dorsal des valves est très incliné dans sa moitié postérieure.

La moitié distale de la furca présente des fines épines. La griffe antérieure de la furca représente environ $75 \%$ de la longueur du bord antérieur du rameau furcal.

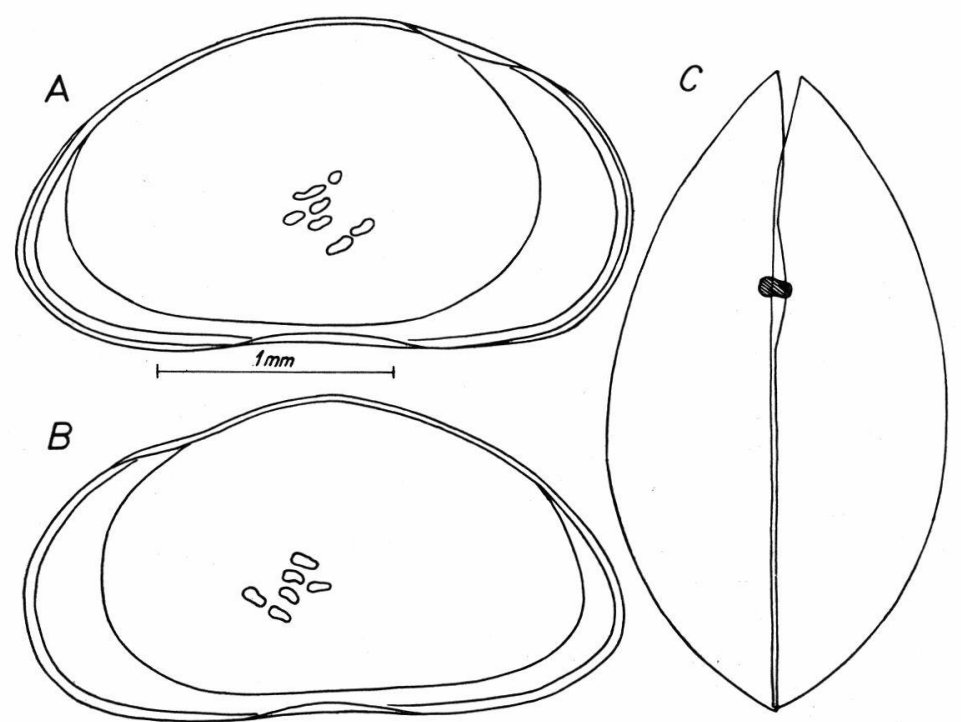

FIG. 2. - Eucypris petkovskii n. sp. ‥ A : valve gauche; $\mathrm{B}$ : valve droite; $\mathrm{C}$ : coquille.

1. C'est pour moi un plaisir de dédier cette nouvelle espèce au spécialiste yougoslave M. T. PETKovski en signe de reconnaissance et vive gratitude. 


\section{Description.}

Coquille (fig. 2 C). - Forme ovale, pointue aux extrémités antérieure et postérieure. La plus grande largeur se trouve dans la moitié postérieure. Couleur vert foncé.

La valve gauche dépasse légèrement la droite.

La longueur des valves mesure entre 2,2 et $2,7 \mathrm{~mm}$.

Valve gauche (fig. $2 \mathrm{~A}$ et fig. $10 \mathrm{~A}$ ). - Bord dorsal fortement courbé, rejoignant le bord antérieur et le bord postérieur sans transition. Son point le plus élevé se trouve en arrière du tiers antérieur (à $45 \%$ de la longueur). A partir de ce point, sa pente est plus raide vers l'arrière que vers l'avant. Le bord postérieur

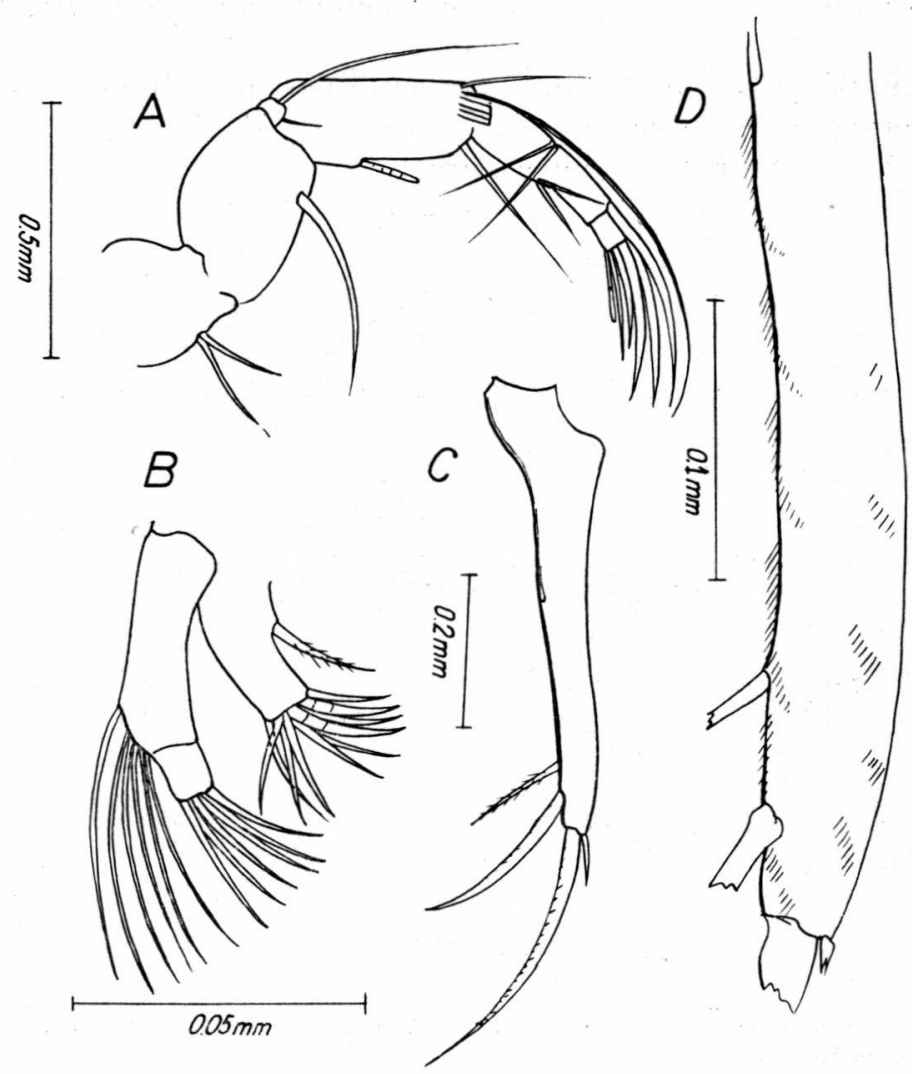

Fig. 3. - Eucypris petkovskii n. sp. + . A : 2 antennes; B : maxille (détail); C : furca; D : furca (détail). 
est plus petit que le bord antérieur. Le contour apparent de la valve, du côté ventral, est presque rectiligne.

La lamelle hyaline est visible au bord antérieur et à celui postérieur.

Valve droite (fig. $2 \mathrm{~B}$ et fig. $10 \mathrm{~B}$ ). - Le bord dorsal forme un angle à son point le plus élevé, au 48-50\% de la longueur. Vers l'avant, dans le tiers antérieur, le bord dorsal forme une inflexion. Vers l'arrière, il rejoint le bord postérieur dans une pente plus inclinée que celle de la valve gauche. Le bord ventral présente une inflexion dans sa partie médiane.

Deuxième antenne $(f i g .3 \mathrm{~A})$. - Les 5 premiers poils natatoires atteignent l'extrémité distale des griffes. Le $6^{\mathrm{e}}$, plus court, dépasse à peine la moitié de l'avant dernier article.

Mandibule (fig. 6 B). - L'extrémité masticatrice porte 7 dents et 2 poils longs et pennés. Entre la première et la deuxième dent existent 2 fortes épines pectinées sur leur tiers distal. Entre la deuxième et la troisième dent se trouvent aussi une épine pectinée ainsi que 2 poils longs; entre les autres dents, 5-7 poils courts.

Maxille (fig. $3 \mathrm{~B}$ ). -- Les griffes de l'endite distal ne sont pas dentelées.

La griffe distale dépasse de deux tiers la longueur de l'endopodite.

Furca (fig. $3 \mathrm{C}, \mathrm{D}$ ). - Le bord postérieur présente une inflexion dans la moitié supérieure. La moitié distale présente de fines épines, très évidentes sur le bord postérieur, où on aperçoit 6 groupes distincts, dont 4 avant le poil postérieur.

Les 2 griffes distales sont finement pectinées sur le bord postérieur. La griffe antérieure représente $75 \%$ de la longueur du bord antérieur de la furca. La griffe postérieure atteint $66 \%$ de la griffe antérieure.

Le poil postérieur a une longueur double de celle de son homologue antérieur; de plus, il est finement barbelé.

\section{Mâle inconnu.}

Affinités. - La forme de la coquille, vue de dos, rapproche $E$. petkovskii n.sp. de E. ornata (O. F. Muller), et E. crassoüdes Ám. Le point le plus élevé des valves est situé chez $E$. ornata et $E$. crassoüdes près du tiers antérieur de la longueur, le bord dorsal dans la moitié postérieure étant peu incliné; chez notre espèce, le point le plus élevé est situé près de la moitié de la longueur, la pente du bord dorsal étant plus raide dans la moitié postérieure. De plus, chez $E$. ornata, la griffe antérieure atteint à peu près la longueur du bord antérieur de la furca, tandis que chez notre espèce la même griffe ne dépasse pas $3 / 4$ de la longueur de la furca. 
Je tiens à remarquer que Bronstein [1947] n'a pas raison quand il considère $E$. crassoïdes comme étant le juvénile d'E. crassa, car la présence de l'appareil copulateur et des organes préhenseurs mâles, figurés par ALM [1914], sont des caractères qui apparaissent seulement chez l'adulte.

La présence des fines épines sur la furca, ainsi que le rapport entre la longueur du bord antérieur de la furca et la griffe antérieure, pourrait rapprocher E. petkovskii n.sp. d'E. luttaria (Косн) et d'E. kerkyrensis STEPhanides.

La longueur des poils natatoires, ainsi que la forme des valves, différencient nettement les 3 espèces, car $E$. luttaria présente des poils natatoires qui ne dépassent pas le dernier article, le bord dorsal des valves étant très peu incliné.

Chez E. kerkyrensis, la hauteur du bord postérieur de la valve est à peu près égale à celle du bord antérieur.

Eucypris pigra pigra (Fischer, 1851).

Matériel : 5 q̊ récoltées par MM. Fr. Botea et C. Plesa dans un sondage effectué dans la «Valea Poicului» (Monts Apuseni, Reg. Cluj), 7.VI.1965, 1 \& récoltée par $\mathbf{M}^{\text {me }}$ E. Prunescu-Arion dans la source de la rivière Moldova (Reg. Maramures), 24.X.1963, et 3 ㅇ dans la rivière Moldova, près de la localité Plaiul Moldovei (Reg. Suceava), leg. E. Prunescu-Arion, 11.VIII.1963.

Les poils natatoires de la deuxième antenne sont très réduits; ils atteignent la moitié de la longueur de l'avant dernier article.

Heterocypris maura (Masi, 1932).

Matériel : 2 qq récoltées par M. C. Plesa dans une mare près du lac Tekirghiol (Reg. Dobrogea), 25.VI.1958.

Je suis d'accord avec KuIE [1937] et Petrovski [1964] pour considérer cette espèce comme appartenant au genre Heterocypris Claus, car elle ne possède pas la protubérance caractéristique du genre Cyprinotus Baird.

La valve gauche (fig. $4 \mathrm{~A}$ ) des deux exemplaires de Heterocypris maura examinés a une forme triangulaire. Leurs longueurs ne dépassaient pas $1,05 \mathrm{~mm}$.

\section{2) Tribu Dolerocypridini.}

Dolerocypris sinensis Sars, 1903.

Matériel : 3 \&o recueillies par le Prof. N. Botnariuc dans les bassins des rizières de Chirnogi (Reg. Bucureşti), 22.VI.1963.

Cette espèce est facilement reconnaissable grâce à la longueur des valves qui atteignent $2,1 \mathrm{~mm}$. 


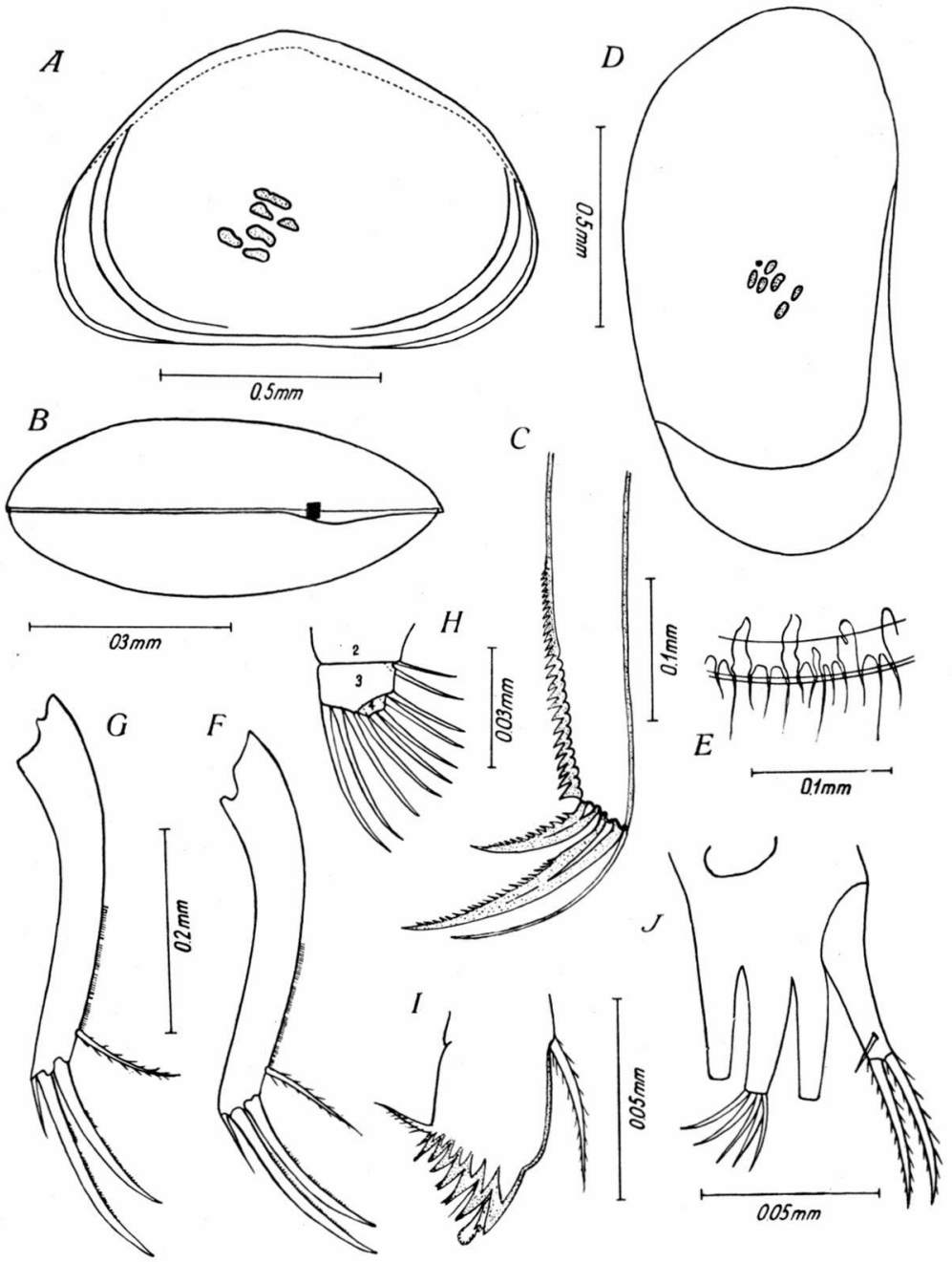

FIG. 4. - A : Heterocypris maura (MASI) + , valve droite; B : Cypridopsis subterranea Wolf o, coquille; C : Stenocypris major (BaIRD), furca (détail); D-G : Isocypris arnoldi Dubowski o. D : valve droite; $\mathrm{E}$ : valve droite (détail du bord antérieur); $F$ : furca droite; G : furca gauche; H-J : Metacypris cordata BR. et RoBert o. H : palpe mandibulaire (détail); I : mandibule (détail); J : maxille. 
3) Tribu Herpetocypridini.

Herpetocypris chevreuxi (Sars, 1896).

Matériel : nombreux exemplaires $\$ q$ et juvéniles récoltés par D. Dancau, le 4.X.1964, dans un petit ruisseau du Jardin Botanique de Bucarest.

La deuxième antenne possède des poils natatoires qui atteignent l'extrémité distale des griffes.

Je suppose que JaNCARIK [1952] a raison quand il considère $H$. palpiger Lowndes [1932] comme synonyme de $H$. chevreuxi Sars.

Isocypris arnoldi Dubowski, 1927.

Matériel : 2 qo récoltées le $30 . X .1964$ dans une mare près de Brăila (dans la proximité de la localité Vărsătura Reg. Galaţi).

Cette espèce a été décrite par Dubowski [1927] d'après un seul exemplaire récolté près de Harcow (Sud de l'U.R.S.S.).

Nos exemplaires diffèrent par la plus grande longueur des valves (fig. $4 \mathrm{D})(1,35-1,4 \mathrm{~mm}$ au lieu de $1,15 \mathrm{~mm})$, et par l'étendue de la région pectinée de la furca : dans le dessin de Dubowski, les deux tiers à peu près du bord dorsal de la furca sont munis de fines épines, tandis que, chez nos exemplaires, elles atteignent seulement la moitié.

Cette espèce fut contestée par Redeke et Den-Dulke [1940] qui la considéraient comme un synonyme de I. prionema G. W. Muller.

Isocypris arnoldi diffère nettement de cette dernière espèce par l'absence de la lamelle hyaline du bord antérieur des valves.

I. arnoldi se rapproche de 1 . beauchampi PARIS par la symétrie des furcas et diffère d'I. quadrisetosa Rome, dont la furca droite est moins arquée que la gauche.

La furca légèrement arquée distingue d'une manière évidente l'espèce de Dubowski d'I. beauchampi.

Stenocypris major (Baird, 1859).

Matériel : 6 \% récoltées par le Prof. N. Botnariuc, le 22.VI.1963, dans les rizières de Chirnogi (Reg. Bucureşti).

Récemment, Rome [1961] a montré l'importance systématique du nombre des dents, ainsi que de leur forme et l'arrangement sur la furca droite.

Chez les exemplaires examinés, la longueur de la valve atteint $2 \mathrm{~mm}$ et la furca droite a 27 dents (fig. $4 \mathrm{C}$ ).

Fergusson [1964] a créé pour le genre Stenocypris une nouvelle sous famille - Stenocyprinae -. 
Les affinités du genre Stenocypris avec Acocypris, Herpetocypris, Isocypris ne laissent aucun doute sur le fait qu'ils représentent une seule lignée phylétique.

Il existe aussi une asymétrie de la furca chez Isocypris quadrisetosa Rome et l'absence du poil postérieur de la furca est propre aussi au genre Acocypris. Donc je considère qu'on ne peut pas établir une nouvelle sous-famille en partant de ces caractères.

\section{C. - Sous-famille CYPRIDOPSINAE Kaufmann, 1900.}

Cypridopsis subterranea Wolf, 1919.

Matériel : 8 우 récoltées par $\mathrm{M}^{\mathrm{me}}$ E. Prunescu-Arion, le 24.X.1963, dans la source de la rivière Moldova (Reg. Maramureş).

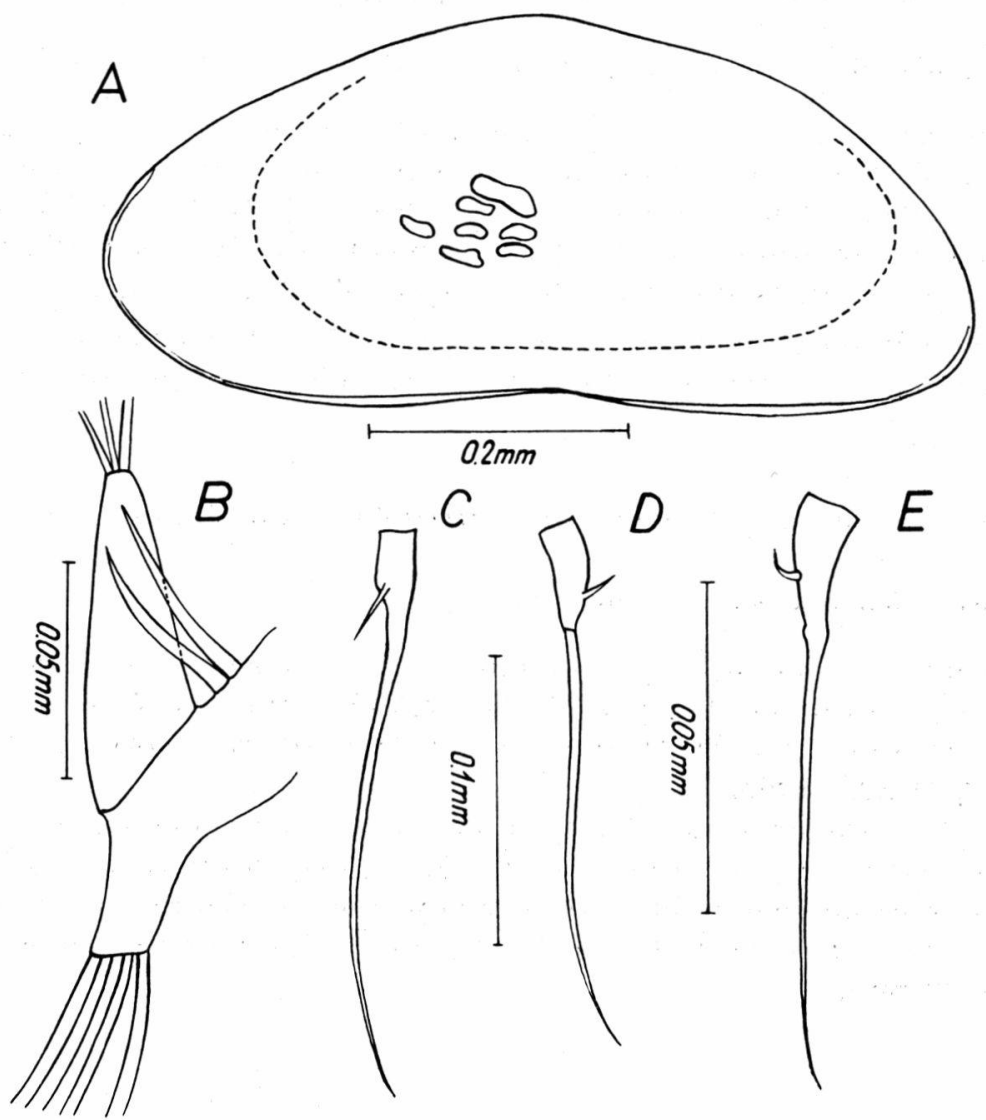

FIG. 5. - A-C : Cypridopsis subterranea Wolf o. A : valve gauche; B : $1^{\text {re }}$ patte (détail); C : furca; D : Potamocypris arcuata (SARs) ${ }^{\circ}$, furca; E : Potamocypris wolfi o, furca. 
Chez les exemplaires étudiés, l'extrémité postérieure de la coquille (fig. $4 \mathrm{~B}$ ) est allongée.

'La valve gauche (fig. $5 \mathrm{~A}$ ) atteint $0,65 \mathrm{~mm}$; la plaquette branchiale de la première patte (fig. $5 \mathrm{~B}$ ) présente seulement 2 rayons; le flagelle de la furca ( $f i g .5 \mathrm{C}$ ) est 5 fois plus long que le rameau furcal.

Potamocypris arcuata (Sars, 1903).

Matériel : 5 ㅇ récoltées le 30.X.1964 dans la même mare qu'Isocypris arnoldi et Eucypris petkovskii.

Cette espèce est facilement reconnaissable par le contour des valves, la longueur des poils natatoires, qui dépassent l'extrémité distale des griffes, et la longueur du flagelle de la furca (fig. $5 \mathrm{D}$ ).

Le nombre trop réduit d'exemplaires ne m'a pas permis d'aborder le problème de la variabilité des valves de $P$. arcuata.

Potamocypris wolfi Brehm, 1920.

Matériel : 2 exemplaires $\%$, dont l'un récolté par $\mathbf{M}^{\text {me }}$ G. PalaDIAN, le 28.IV.1964, dans la rivière Teleajen près de la localité Cheia (Reg. Ploieşti), l'autre par C. PlesA, le 27.IX.1961, dans un sondage phréatique effectué au confluent des rivières Cîrligaţi et Drăgan, affluents du Criş (Monts Apuseni, Reg. Cluj).

Le poil postérieur de la furca (fig. $5 \mathrm{E}$ ) est courbé à $90^{\circ}$. Le flagelle de la furca est 4 fois plus long que le rameau furcal.

D. - Sous-famille CYCLOCYPRIDINAE Kaufmann, 1900.

Cypria lacustris Sars, 1890.

Matériel : nombreux exemplaires $\varsubsetneqq \uparrow$ et juvéniles récoltés dans un puits situé sur la plage de la localité 2 Mai (près du bord de la Mer Noire) leg. D. Danielopol, D. Dancau, E. Serban, I. TabaCARU, le 20.X.1963.

Les exemplaires que j'ai examinés possédaient une seule expansion digitiforme sur le lobe génital. Le coloris des valves était brun clair, avec une bande jaunâtre sur la partie antérieure. La longueur des valves était de $0,58 \mathrm{~mm}$, tandis que la hauteur moyenne était de $0,39 \mathrm{~mm}$. 
II. - FAMILLE CYTHERIDAE Baird, 1850.

Sous-famille LIMNOCYTHERINAE Sars, 1925.

En résumant les principaux caractères de cette famille, SARS [1925] dit : «Besides, by their confinement to fresh water, the forms comprised within the present subfamily distinguish themselves from other Cytheridae by the thin corneous shell, the imperfectly developed hinge, and the presence of only a single eye, as in most of the Cypridae » (p. 149).

De plus, il ajoute page 148 : "Both pairs of antennae distinctly geniculate and subpediforme ».

SARS et, plus tard KLIE [1938] n'ont pas raison quand ils considèrent comme un caractère de sous-famille la structure tri-articulée du palpe de la mandibule, car chez Gomphocythere crista RoME, ainsi que chez Metacypris cordata Br. et RoвErT (fig. $4 \mathrm{H}$ ), le palpe comprend 4 articles distincts.

En dehors du genre Pseudolimnocythere KuIE, 1938 dont la position systématique reste à résoudre, tous les genres de cette sous-famille possèdent 3 griffes à l'extrémité distale de la deuxième antenne.

Vu la structure de l'extrémité distale de la première antenne, ainsi que celle du palpe maxillaire, on peut entrevoir deux lignées phylétiques distinctes.

D'une part, on peut grouper les genres dont l'extrémité distale de la première antenne possède le poil sensitif en forme de massue, soudé par sa base à un des poils adjacents (fig. $8 \mathrm{~J}$ ), et le palpe de la maxille divisé en deux articles, dont chacun est pourvu au bord distal d'un nombre variable de poils qui existent même si ces deux articles sont soudés (fig. $8 \mathrm{~K}$ ). D'autre part, il y a les genres dont l'extrémité distale de la première antenne possède le poil sensitif en forme de massue, libre (fig. $8 \mathrm{~A}$ ), et le palpe de la maxille formé d'un seul article, pourvu seulement à l'extrémité distale de 2 à 4 gros poils pennés (fig. $4 \mathrm{~J}$ ).

J'ai nommé la première lignée, tribu Limnocytherini, d'après le genre type de la sous-famille des Limnocytherinae.

Outre ce genre, j'ai examiné aussi des exemplaires de Gomphocythere SARs et Cytheridella DADAY qui possèdent eux aussi ces caractères.

Dans la deuxième lignée, nommée tribu Metacyprini nov. d'après le genre Metacypris BR. et RoBERT., on doit grouper, outre le genre type, les genres Aphrocythere KLIE, 1935, Elpidium F. Muller et Cordocythere n. g. 


\section{1) Tribu Limnocytherini nov.}

Lymnocythere inopinata (Baird, 1843).

Matériel : 8 우 récoltées dans une mare près du Lac Sărat ('Reg. Galați), le 7.VII.1964.

Les exemplaires étudiés ne possédaient pas de tubérosités sur les valves.

La valve gauche présentait sur le bord ventral postérieur un nombre variable de dents (3-4 en moyenne), dont la taille diminue vers le bord postérieur.

L'extrémité masticatrice de la mandibule (fig. $6 \mathrm{C}$ ) porte 7 dents, une courte épine et un poil long et penné.

Entre la première et la deuxième dent, ainsi qu'entre cette dernière et la troisième, il existe une forte épine dont l'extrémité distale n'est pas pointue, et un poil long. Des poils sensoriels courts existent aussi entre les autres dents.

2) Tribu Metacyprini nov.

Metacypris cordata Brady et Robertson 1870.

Matériel : 12 exemplaires juvéniles (dans le dernier stade) récoltés le 21.X.1964, en compagnie de I. TABAcarU, dans le Lac Căldăruşani (Reg. Bucureşti).

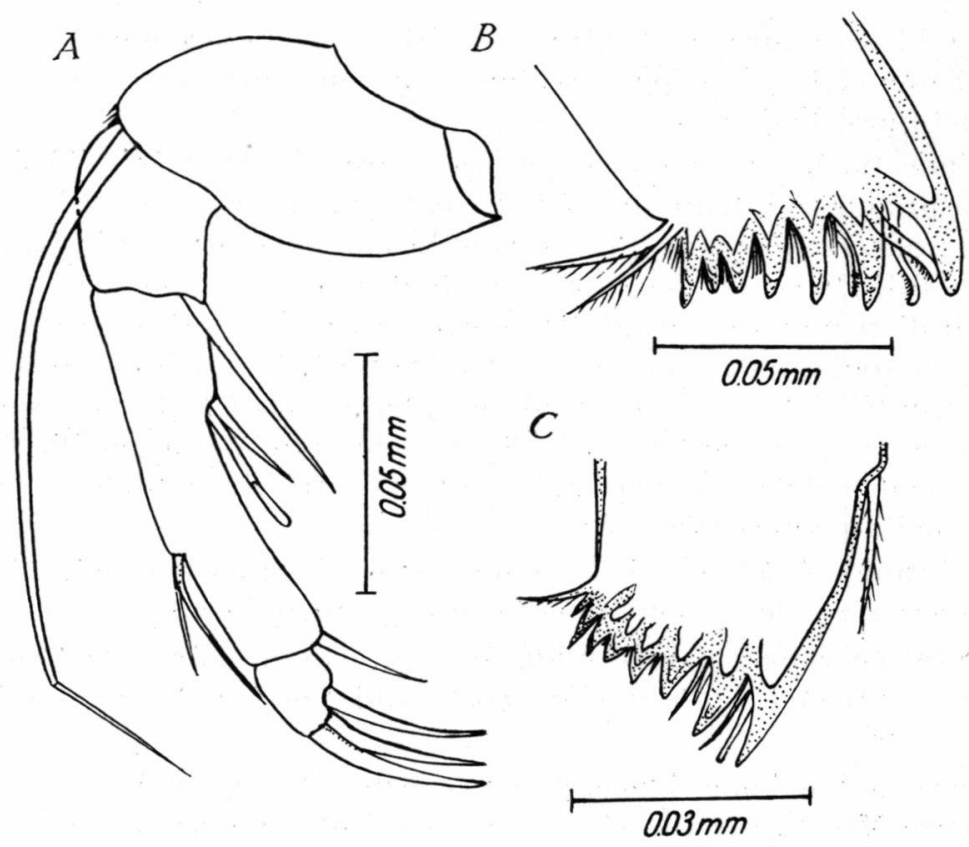

FIG. 6. - A : Metacypris cordata BR. et RoBert o, $2^{e}$ antenne; B : Eucypris petkovskii n. sp. o, mandibule (détail); $\mathrm{C}^{+}$: Limnocythere inopinata (BAIRD) + mandibule (détail). 
Les valves des exemplaires que j'ai récoltés à Căldăruşani mesuraient entre 0,42 et $0,45 \mathrm{~mm}$.

Par contre, les 4 valves envoyées par le Dr E. Triebel mesuraient $0,52-0,55 \mathrm{~mm}$. Le fait que chez les exemplaires roumains l'orifice génital femelle, chitinisé, était absent (il a été figuré par Pony I [1962] chez des exemplaires adultes du lac Balaton) me fait supposer que mon matériel était composé seulement par des juvéniles au dernier stade.

Les valves des adultes, envoyées par le Dr E. Triebel, provenaient d'un dépôt holocène d'Arnstadt (Allemagne). Leurs replis se trouvaient très à l'intérieur de la valve (fig. $9 \mathrm{~A}$ ) spécialement dans la région ventro-postérieure, la charnière possédait sur la valve droite une dent antérieure de forme lamellaire, non divisée. Sur la valve gauche, une fossette antérieure (fig. $7 \mathrm{H}$ ) copie la forme de la dent opposée.

Chez les exemplaires de Căldăruşani, Ie repli était moins développé et la dent antérieure de la valve droite, de forme lamellaire, était finement divisée en 3-4 cuspides. La fossette de la valve gauche (fig. $9 \mathrm{~B}$ ), en copiant la forme de la dent opposée, était pourvue de 3-4 alvéoles.

Dans ce qui suit, je vais décrire les principaux appendices des exemplaires roumains.

Deuxième antenne (fig. $6 \mathrm{~A})$. - Elle présente 2 poils sur le bord dorsal de l'avant-dernier article. G. W. Müller [1900] figure un seul poil pour les exemplaires d'Allemagne.

Mandibule (fig. 4I). - J'ai remarqué 7 dents lamellaires, dont les premières ont chacune 3 pointes. La septième ayant une forme allongée, comporte probablement seulement 2 pointes. Près de cette dernière dent, il existe une courte épine et un long poil penné.

Entre la première et la deuxième dent, il existe une épine spatulée et crénelée dans sa moitié distale. Il est très probable qu'il existe aussi un poil long que je n'ai pas pu apercevoir avec précision, vu les dimensions réduites de la pièce et les difficultés de la préparation.

Entre la deuxième et la troisième dent il existe un poil long et mince.

W. Tressler [1941], dans un tableau comparatif concernant les espèces du genre Metacypris, considère que Metacypris cordata aurait « 4-5 dents ayant chacune deux ou plusieurs pointes» (p. 265 ).

A mon avis le nombre des dents de la mandibule est un caractère constant. En comparant la forme de la mandibule chez des espèces éloignées du point de vue phylétique, Limnocythere inopinata (BAIRD) (fig. $6 \mathrm{C}$ ) et Eucypris petkovskii n. sp. (fig. $6 \mathrm{~B}$ ), j'ai constaté qu'elles présentaient de même 7 dents, mais que le nombre 
et la forme des épines et des poils qui se trouvent entre les dents variaient.

Palpe mandibulaire (fig. $4 \mathrm{H}$ ). - Il est formé par 4 articles. KLIE [1938] et Bronstein [1947] considèrent que ce palpe est formé chez l'adulte de 3 articles, dont le dernier provient de la fusion de 2 articles.

Chez les exemplaires roumains que j'ai étudiés au microscope à contraste de phase, j'ai observé une délimitation précise entre le troisième et le quatrième article. Ce dernier est très réduit et possède de longs poils.

Sur la moitié distale du bord externe de l'avant dernier article sont insérés 3 longs poils. Sur le bord interne du deuxième article il y a 2 poils.

Maxille $(f i g .4 \mathrm{~J})$. - L'endite moyen de la maxille présente 5 épines, dont 2 plus fortes. Chez les exemplaires étudiés par J. P. Wolf [1915], le nombre des épines de cet endite variait de 2 à 4 . Je n'ai pas remarqué pour ma part une telle variabilité.

Plaquette branchiale (fig. $9 \mathrm{D}$ ). - Elle possède un poil aberrant placé légèrement à l'intérieur.

Palpe maxillaire (fig. $4 \mathrm{~J}$ ) court, il est formé d'un seul article qui possède à l'extrémité distale 2 forts poils pennés et un poil court placé à l'intérieur.

Furca (fig. $9 \mathrm{C}$ ). - Elle possède un lobe avec 3 expansions poilues qui s'amincissent vers le bout.

Cordocythere n. g.

Diagnose. - Genre appartenant à la tribu Metacyprini, caractérisé par la forme des valves qui possèdent un sillon médian transversal. Chez les femelles, la coquille, dans la moitié postérieure, est fortement développée et transformée en cavité incubatrice.

La charnière sur la valve gauche possède une dent lamellaire antérieure, divisée en 3-4 cuspides et une forte dent postérieure, incurvée.

La furca possède 3 expansions, dont la postéro-inférieure, digitiforme, est très gonflée à la base.

Le nom a été donné d'après la forme de la coquille vue de dos, qui ressemble à un cœur. (Du latin cor-cordis, le cœur.)

L'espèce type est Cordocythere phreaticola n. sp.

Cordocythere phreaticola n. sp.

Locus typicus - la grotte de Vadu-Crişului (la grotte de Zichy), dans les interstices d'une plage de gravier fin, placée dans la zone du premier siphon (fig. 11). 
Matériel : 5 exemplaires 1958), 2 ㅇ, 1 juv. (le même endroit, 21.X.1959), 1 , 5 juv. (le même endroit, 28.VI.1961), 2 \&̊ 4 juv. (même endroit, 30.VIII.1961), 5 juv. (même endroit, 7.XI.1961), leg. C. Plesa, 3 \&̊ (même endroit, 6.X.1961), leg. C. Plesa et D. Dancau.
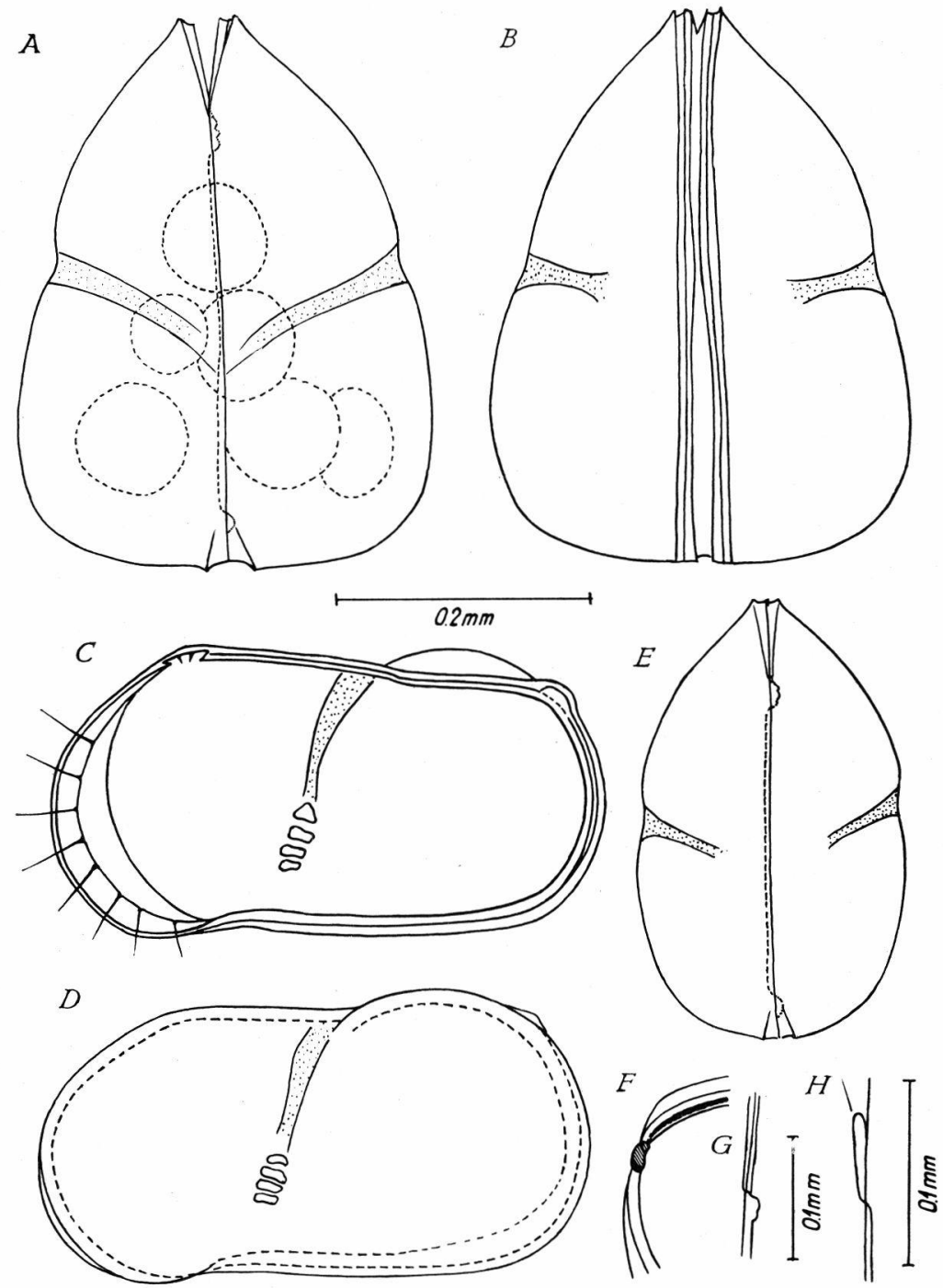

Fig. 7.- A-G : Cordocythere phreaticola n. g. n. sp. A : coquille vue de dos; B : coquille vue du côté ventral; $\mathrm{C}$ : valve droite, vue latérale interne; D : valve gauche vue latérale externe; $\mathrm{E}$ : exemplaire juvénile, coquille vue de dos; F : valve gauche (détail); G : valve droite (détail); $\mathrm{H}$ : Metacypris cordata Br. et RoBert o (exemplaire d'Arnstadt) fossette antérieure, valve gauche. 
On a également trouvé cette espèce dans des sondages phréatiques exécutés sur une plage de la rivière Misid, à $100 \mathrm{~m}$ en aval de la grotte Moana (3 $q$, 19.VIII.1963) leg. C. Plesa, F. BoteA, G. Racovita; ainsi que sur une plage de la rivière Cărmăzane (affluent du Misid) sondage phréatique d'une plage de gravier (1 juv., 18.VIII.1963) leg. G. Racovita.

Matériel étudié : 7 ex. $¥$ disséqués, 2 ex. juv. Note : 3 exemplaires femelles non disséqués ont été déposés au British Museum (Natural History) sous le numéro B.M. 1965. 4.5.2.

\section{Description.}

Coquille. - Vue de dos (fig. 7 A), elle est dilatée dans la moitié postérieure. Le sillon médio-transversal des 2 valves s'unit par le bord dorsal aux $3 / 5$ de la longueur.

Par transparence, on aperçoit la charnière et la cavité incubatrice. Cette dernière peut contenir 3 à 6 œufs. Vu du côté ventral (fig. $7 \mathrm{~B}$ ), le sillon médio-transversal ne partage pas complètement les valves.

$\begin{array}{cccc}\text { Dimensions des valves : } & \text { longueur } & \text { hauteur } & \text { largeur } \\ \text { valve gauche } & \mathbf{0 , 4 0 4} \mathrm{mm} & 0,204 \mathrm{~mm} & 0,305 \mathrm{~mm} \\ \text { valve droite } & \mathbf{0 , 4 0 8} \mathrm{mm} & \mathbf{0 , 2 0 4} \mathrm{mm} & \end{array}$

Valve droite (fig. $7 \mathrm{C}$ et $10 \mathrm{C}$ ). - Vue de côté, elle présente un sillon médio-transversal peu profond, qui se perd dans la région ventrale.

Le bord dorsal est droit, à l'exception de la portion du sillon médian.

Le passage vers le bord postérieur est brusque, à angle presque droit, tandis que le passage du bord postérieur vers le bord ventral est nettement courbé. Le bord ventral est droit dans sa portion médiane. Le passage vers le bord antérieur, largement courbé, se fait par une inflexion. Entre le bord dorsal et le bord antérieur existe un angle d'environ $120^{\circ}$.

Le repli est développé dans la région ventrale et postérieure.

Valve gauche (fig. $7 \mathrm{D})$. - Elle possède sur le bord antérieur une courte lamelle hyaline, qui manque à la valve droite. De même que sur la valve droite, les impressions musculaires, au nombre de 4, disposées en ligne droite, sont placées dans la région du sillon médian (fig. $10 \mathrm{D}$ ).

Le repli est plus développé que sur la valve droite.

Charnière (fig. $7 \mathrm{~A}, \mathrm{G}$ ). - La valve droite possède sur le bord dorsal 2 fossettes ouvertes, celle antérieure ayant à l'intérieur 3-4 alvéoles incomplètement séparées. La fossette postérieure est à peine divisée. Entre ces deux fossettes se trouve une fine lamelle médiane. 
La valve gauche possède une dent lamellaire antérieure, dont le bord externe est divisé en 3-4 cuspides et une forte dent incurvée, placée à l'angle dorso-postérieur. Entre ces deux dents, il y a un sillon peu profond dans lequel s'encastre la lamelle médiane de la valve opposée.

Ornementations (fig. $10 \mathrm{D}$ ). - La surface externe des valves présente de nombreuses alvéoles.

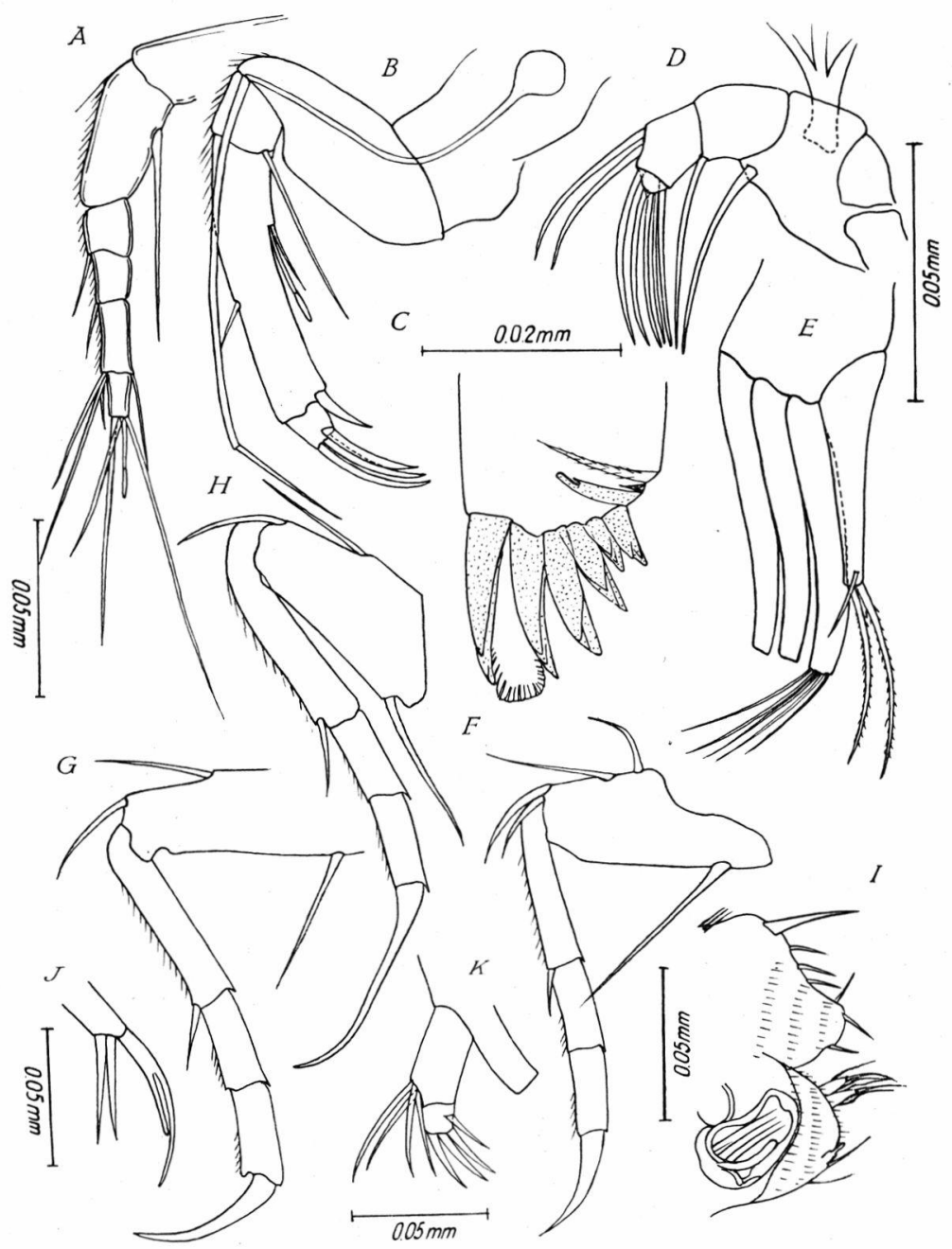

Fig. 8. - A-I : Cordocythere phreaticola n. g. n. sp. 9, A : $1^{\text {re }}$ antenne; $\mathrm{B}: 2^{\mathrm{e}}$ antenne; $\mathrm{C}:$ mandibule (détail); D : palpe mandibulaire; $\mathrm{E}$ : maxille (détail); F : $1^{\text {re }}$ patte; G : $2^{\mathrm{e}}$ patte; H : $3^{\text {e }}$ patte; I : extrémité postérieure du corps et furca; J-K : Limnocythere inopinata (BAIRD), J : $1^{\text {re }}$ antenne (détail); $\mathrm{K}$ : palpe maxillaire. 
La couleur des exemplaires conservés en alcool $70^{\circ}$ est grise blanchâtre.

Première antenne (fig. $8 \mathrm{~A}$ ). - Elle est formée de 6 articles dont la longueur est dans le rapport suivant : 17:18:5:6:8:5.

Sur le bord antérieur, sont insérés un poil à chaque extrémité distale des articles 3 et 4 , et 3 poils de longueurs différentes à l'article 5. Sur le bord postérieur, il existe un long poil inséré à l'article 2, et un poil plus court inséré à l'article 5. A l'extrémité distale de l'article 6 sont insérés 3 poils de différentes longueurs, le médian étant le plus court, et un poil sensitif en forme de massue, qui n'est pas soudé par sa base à un autre poil.

Deuxic̀me antenne (fig. $8 \mathrm{~B}$ ). - Elle possède un poil formé de 2 segments; la longueur du premier atteint l'extrémité distale du troisième article de l'antenne. Sur le bord antérieur de ce dernier article, j'ai pu observer un seul poil mince. Sur le bord postérieur de l'extrémité distale de l'article 2 se trouve un poil qui dépasse de deux tiers la longueur de l'article suivant. Dans le tiers supérieur de ce dernier se trouvent insérés un poil court et un poil sensitif, dont la longueur dépasse le poil adjacent. A l'extrémité distale du même article, on distingue facilement une épine épaissie, en forme de griffe. Sur le dernier article se trouvent 3 griffes.

Mandibule (fig. $8 \mathrm{C}$ ). - Elle possède à l'extrémité distale 7 dents lamellaires, dont les 6 premières ont 3 pointes et la septième seulement 2 pointes. A leur suite se trouvent encore une courte épine et un poil penné.

Entre la première et la deuxième dent est insérée une épine spatulée et crénelée dans sa moitié distale.

Palpe mandibulaire (fig. $8 \mathrm{D})$. - - Il est formé de 4 articles, dont la longueur est dans le rapport suivant : 10:9:8:2. Sur le premier article s'insère à l'extérieur une plaquette branchiale formée de 3 rayons.

Sur le bord intérieur, à l'extrémité distale de ce même article, se trouve un long poil. Sur l'article 2 , il y a un seul poil situé du côté interne et distal. Le bord extérieur de l'article suivant forme un angle évident; sur sa portion distale s'insèrent 2 épines.

Maxille (fig. $8 \mathrm{E}$ ). - Elle est formée d'un corps sur lequel s'encastrent les 3 endites masticateurs, terminés chacun par 5 épines.

Plaquette branchiale (fig. $9 \mathrm{E}$ ). - Elle a 15 rayons pennés et un poil aberrant placé légèrement à l'intérieur.

Palpe maxillaire (fig. $8 \mathrm{E}$ ). - Il représente deux tiers de la longueur de l'endite masticateur. A l'extrémité distale, il possède deux longs poils massifs et pennés et un poil court, inséré légèrement à l'intérieur.

Pattes. - Les 3 pattes (fig. $8 \mathrm{~F}, \mathrm{G}, \mathrm{H}$ ) présentent les caractères communs à toute la sous-famille Limnocytherinae. 
Extrémité du corps (fig. $8 \mathrm{I}$ ). — Elle est constituée par un lobe postéro-supérieur, sur le dos duquel s'insère une rangée d'épines longues, nettement visibles en vue dorsale. A l'angle que fait le passage de la région dorsale à la région postérieure, une forte épine s'insère sur une petite proéminence. Sur le bord postérieur de ce lobe, on distingue quelques épines qui dépassent l'extrémité distale du lobe.

Dans la région postéro-inférieure se trouvent 2 lobes symétriques, de chaque côté du lobe postéro-supérieur, représentant la furca.

L'orifice génital est fortement chitinisé.

Furca (fig. $8 \mathrm{I}$ et $9 \mathrm{~F}$ ). - Elle possède 2 expansions au bout desquelles se trouve un poil. D'un côté et de l'autre de chaque expansion, dans la région médiane, on voit 2 longs poils qui atteignent à peu près l'extrémité distale du poil médian.

Une troisième expansion, fortement dilatée dans la région basale, présente 1-2 poils qui s'insèrent à la base. J'ai pu distinguer un poil long, situé dans la région de passage du bord postérieur au bord ventral.

L'œil médian est absent.

La description des exemplaires juvéniles fera l'objet d'une étude séparée; nous devons pourtant signaler qu'au dernier stade de développement la cavité incubatrice n'est pas encore développée (fig. $7 \mathrm{E}$ ).

\section{Mâle inconnu.}

Affinités et différences. - M. le Dr T. Peткovski a déterminé comme étant Metacypris cordata des exemplaires récoltés dans quelques sondages phréatiques effectués dans une plage de gravier de la grotte Vadu-Crişului [in Viehman, Plesa, Rusu 19641. Il s'agit probablement de Cordocythere phreaticola n. g. n. sp.

J'ai déterminé moi-même comme Metacypris sp. des exemplaires récoltés dans la vallée du Misid [in Plesa, Botea, Racovita 1964]. Il s'agit de Cordocythere phreaticola n. g. n. sp.

Le nouveau genre que je viens de décrire présente un sillon médio-transversal, ce qui le rapproche des genres Gomphocythere SARs et Cytheridella DADAY.

Il diffère de ce dernier par la présence des dents sur la valve gauche, ce qui le rapprocherait davantage du genre Gomphocythere. Mais la structure du palpe maxillaire, fort réduit, ainsi que la présence du poil en massue de la première antenne, qui est libre, différencie nettement Cordocythere de Gomphocythere.

La structure de la première antenne ainsi que celle du palpe maxillaire rapproche notre genre des Metacypris Br. et RoBERT et Elpidium F. Müller. Ces deux derniers genres ne présentent pas un sillon médian et les dents de la charnière sont situées sur 
la valve droite, au lieu de la gauche. Le repli chez Metacypris cordata est plus développé dans la région postéro-ventrale que chez notre espèce.

L'espèce fossile Metacypris consobrina (JONEs) retrouvée dans les dépôts crétacés d'Amérique et Afrique, selon Pinto et SanguiNETTI [1961], possède un seul sillon médio transversal, et l'orne-

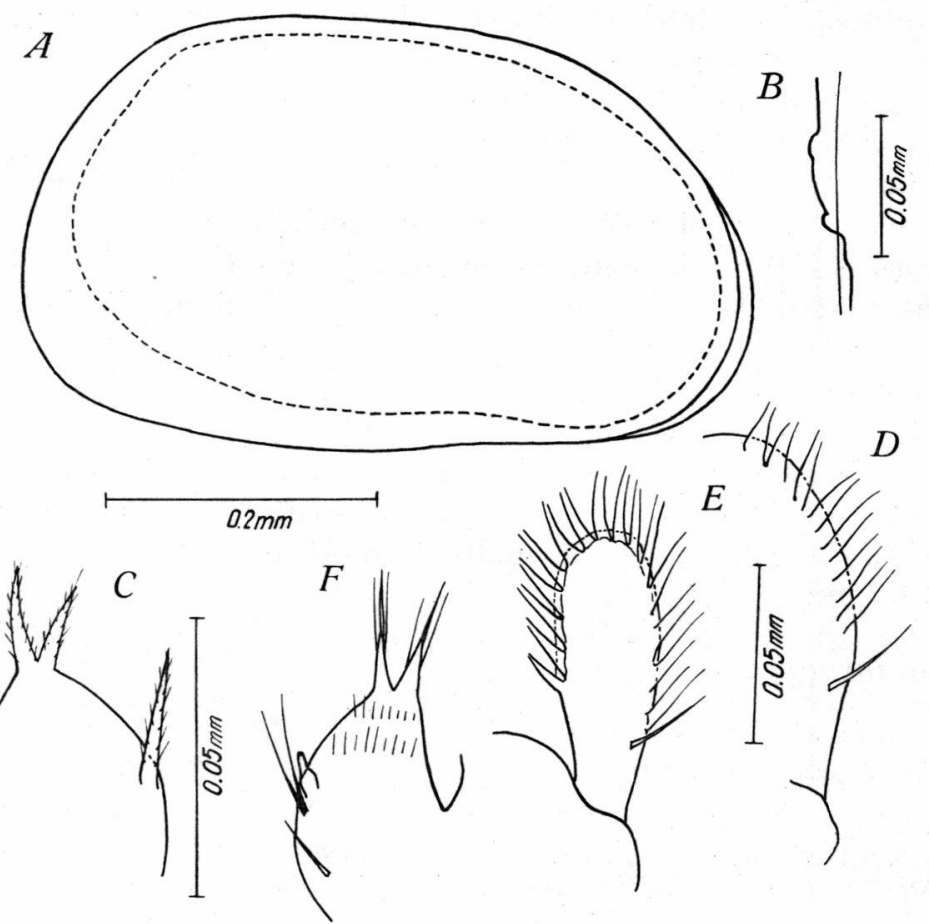

FIG. 9. - A-D : Metacypris cordata Br. et RoBert $\&$ (exemplaire d'Arnstadt). A : valve droite vue latérale externe; $B$ : fossette antérieure de la valve gauche d'un exemplaire roumain; $\mathrm{C}$ : furca; D : plaquettè branchiale maxillaire (détail); E-F : Cordocythere phreaticola n. g. n. sp. E : plaquette branchiale de la maxille; F : furca.

mentation des valves est alvéolaire. Malheureusement, on ne connaît pas la structure de la charnière. Vu la forme extérieure des valves, il n'est pas exclu que cette espèce appartienne au genre Cordocythere $\mathrm{n}$. g.

Même Pinto et Sanguinetri [1961] écrivent (p. 9) «As we do not know if the hingement and the presence of a strong sulcus may have a generic significance, this form may later be referred to a new genus». 


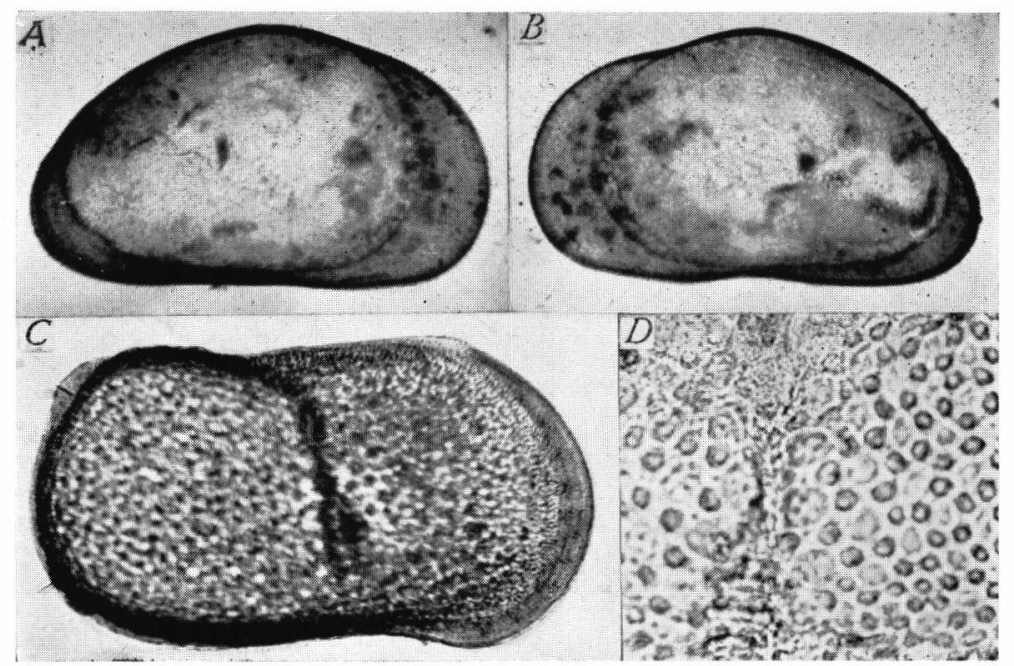

Fig. 10. - A-B : Eucypris petkovskii n. sp. ㅇ. A : valve gauche; B : valve droite; C-D : Cordocythere phreaticola n. g. n. sp. ㅇ․ C : valve droite vue latérale externe; D : valve droite (détail de la région centrale) (photo D. L. Danielopol).

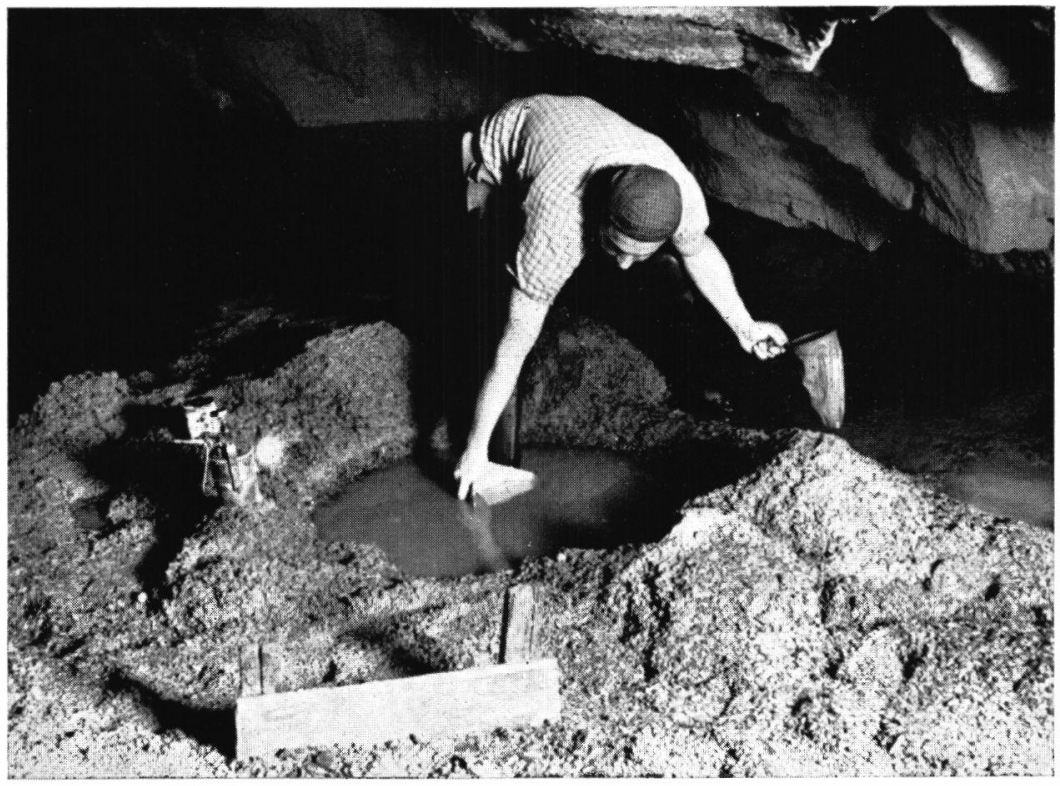

Fig. 11. - Sondage phréatique dans une plage de gravier fin dans la région du premier siphon de la grotte Vadu-Crisului (Photo C. PLesa). 

Le genre fossile Bisulcocypris Pinto et Sanguinetri possède, comme le nom l'indique, 2 sillons transversaux, tandis que Cordocythere n. g. possède un seul sillon.

Vu la structure des valves et de la charnière, le genre Bisulcocypris appartient probablement à la tribu Metacyprini.

\section{REMARQUES ECOLOGIQUES ET ZOOGEOGRAPHIQUES}

La présence des espèces thermophiles Stenocypris major, Dolerocypris sinensis et Heterocypris maura, les 2 premières trouvées dans les rizières de Chirnogi (près de Bucarest), la troisième dans une mare en cours d'assèchement dans le Sud de la Dodrogea, est intéressante du point de vue zoogéographique. Ces stations représentent les limites nord actuellement connues de leurs aires de distribution.

Stenocypris major est une espèce cosmopolite très répandue dans les régions tropicales, Inde, Afrique, Australie, Amérique du Sud et Centrale [Triebel 1953]. Le Dr T. Petrovski nous signale dans une lettre du 25.XII.1964 qu'il a trouvé Iui aussi cette espèce dans les rizières des environs de Skopje.

Puscariu [1951] trouva St. major dans un bassin à Victoria regia, au Jardin Botanique de Cluj. Il l'a considéré comme « une espèce nouvelle pour la faune de la Roumanie ». Toutefois cette espèce, apportée probablement avec les plantes exotiques et maintenue grâce aux conditions spéciales créées au Jardin Botanique,

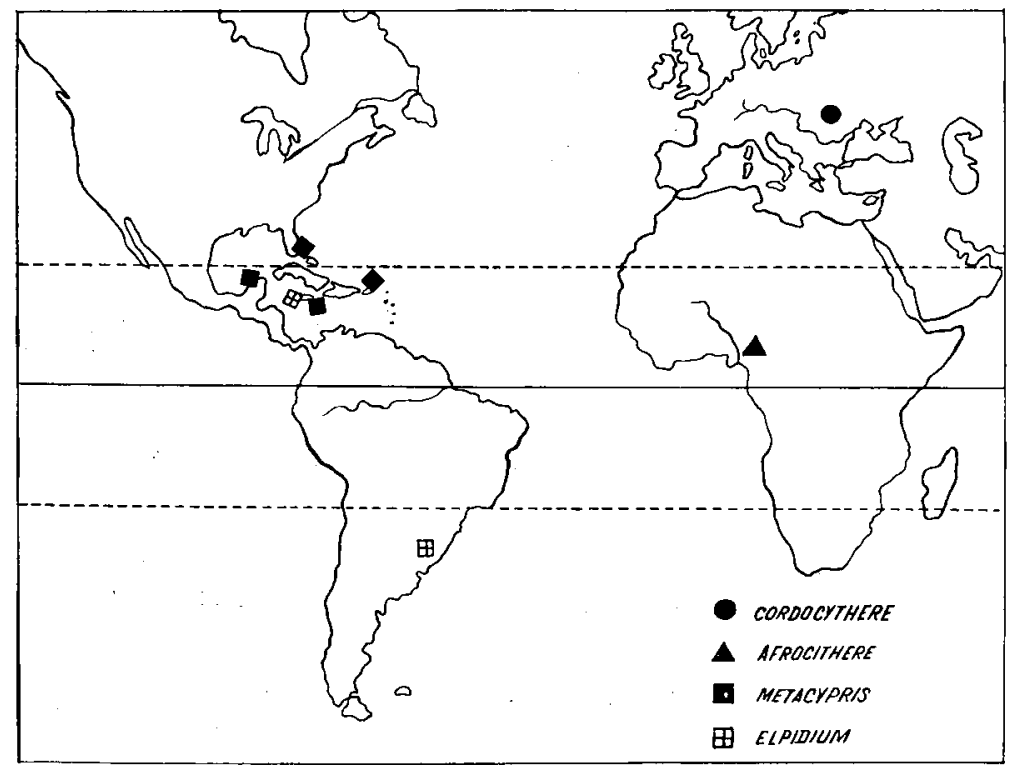

Fig. 12. - Répartition des représentants de la tribus Metacyprini nov. 
ne pouvait pas être considérée à cette date comme appartenant ̀̀ la faune de la Roumanie.

Dolerocypris sinensis et Heterocypris maura sont des espèces d'origine subtropicale. La première a été retrouvée dans le sud de l'Europe : par Bronstein [1947] en U.R.S.S., par CvetKov [1959] en Bulgarie, par Petrovski [1960] en Yougoslavie, par MarazanoF [1965] en France (Camargue); la deuxième a été signalée par Masi [1932] au Maroc, par Klie [1937] en Bulgarie et par PetKovsKi [1964] en Yougoslavie.

LöfFLer [1961] considère que la distribution vers le Nord des Entomostracés d'origine subtropicale est possible seulement dans les régions où il existe une période d'au moins 30 jours ayant une moyenne thermique de minimum $20^{\circ} \mathrm{C}$. Le Sud de la Roumanie représente justement la limite de l'isotherme de cette moyenne thermique.

Isocypris arnoldi, cité jusqu'à présent dans le Sud de l'U.R.S.S. [Duвowsкi, 1927] et de la Roumanie, est remplacé en Europe centrale et occidentale par Isocypris quadrisetosa Rome (Autriche : Lörfler [1963], Allemagne : Herbst [1951], Belgique : RoME [1947], Hollande : Redeke et Den-Dulk [1940]).

La présence de Cypria lacustris dans un puits près du bord de la Mer Noire vient confirmer l'hypothèse de Petkovski [1957] selon laquelle cette espèce serait un élément phréatophile..

Nous signalons pour la première fois la présence de Cypridopsis subterranea en Europe Orientale, d'après ThiEnemann [1950]. Cette espèce est une relicte préglaciaire connue jusqu'à présent seulement en Europe centrale et occidentale (Autriche : Löfrler [1963], Allemagne : Klie [1938], Belgique : Leruth [1939]).

$\mathrm{Vu}$ la répartition actuelle des représentants de la tribu des Metacyprini ${ }^{2}$, Cordocythere phreaticola est à notre avis une relicte préglaciaire (fig. 12).

Metacypris cordata, répandue dans toute l'Europe, est la seule espèce de cette tribu qui a réussi à s'adapter du point de vue physiologique aux rigueurs climatiques propres à la période holocène.

La présence des espèces fossiles Metacypris danubialis danubialis Straub [1952], M. danubialis punctata Straub [1952], et $M$. romboidea Straub [1952], dans le territoire de la Hongrie, prouve que la tribu des Metacyprini était mieux représentée en Europe pendant cette période à climat chaud et constant.

Les échantillons examinés contenant des Cordocythere phreaticola étaient composés seulement d'exemplaires juvéniles et de

2. Metacypris americana Furtos, 1936, M. Maricaœnsis Tressler, 1941, M. Laesslei Tressler, 1956 en Amérique Centrale, Elpidium bromeliarum F. Müller, 1880 en Amérique du Sud et Centrale, Afrocythere rostrata $\mathrm{K}_{\mathrm{LIR}}$ 1935 en Afrique Centrale. 
femelles adultes; la cavité incubatrice de ces dernières contenait 1 à 6 oufs. Ceci me fait supposer qu'il n'existe pas une stricte périodicité de la reproduction.

L'absence des mâles est due probablement à la reproduction de type parthénogénétique.

\section{RÉSUMÉ}

L'auteur présente la description de Cordocythere phreaticola n.g. n.sp., Eucypris petkovskii n.sp. ainsi que des remarques systématiques sur 13 espèces citées pour la la première fois dans la faune de la Roumanie.

On donne la description détaillée de la mandibule et de la maxille de Metacypris cordata, fort utile pour la systématique de la tribu des Metacyprini nov. Une analyse comparative portant sur la structure de l'antenne I, la mandibule et la maxille des genres Limnocythere, Cytheridella, Gomphocythere, Metacypris, Elpidium et Cordocythere a permis à l'auteur d'entrevoir l'existence de deux lignées phylétiques à l'intérieur de la sous-famille des Limnocytherinae Sars 1925. Elles ont été nommées tribu des Limnocytherini nov. et des Metacyprini nov.

La redécouverte d'Isocypris arnoldi, pour la première fois depuis sa description initiale, est intéressante au point de vue systématique et zoogéographique.

La présence des espèces thermophiles Dolerocypris sinensis, Stenocypris major et Heterocypris maura dans le Sud de la Roumanie, ainsi que l'espèce phréatophile Cypridopsis subterranea dans le Nord-Est, est intéressante au point de vue zoogéographique.

Vu la répartition actuelle des représentants de la tribu des Metacyprini, Cordocythere phreaticola est considéré une relicte préglaciaire.

Systematic: and Faunistic notes on Freswater Ostracods of RUMANIA.

The author presents the description in detail of Cordocythere phreaticola n.g. n.sp., Eucypris petkovskii n.sp. and some systematic remarks on 13 species, first mentioned in the fauna of Rumania. He gives the detailed description of the mandibula and maxilla of Metacypris cordata, very useful for the systematic of the Metacyprinii nov. trib.; a comparative analysis concerning the structure of the first antenna, the mandibula and the maxilla of the genus Limnocythere, Cytheridella, Gomphocythere, Metacypris, Elpidium and Cordocythere permited the author to foresee the existance of two phyletic strains inside the Limnocytherinae Sars, 1925 subfamilly. They were named tribus Limnocytherini nov. and tribus Metacyprini nov.

The redescovery of Isocypris arnoldi for the first time since its initial description, is interesting from the systematic and zoogeographic point of view.

The presence of the thermophile species Dolerocypris sinensis, Stenocypris major and Heterocypris maura in the South of Rumania, so as the phreatophile species Cypridopsis subterranea in the North-East, is interesting from the zoogeographical point of view. 
Taking in consideration the actual repartition of the Metacyprini tribus representatives, Cordocythere phreaticola is considered a preglacial relict.

\section{SyStematische UND FAUNistische UNTERSUChungen übER DEN Suss- WASSER-OSTRACOdEN RüManiens.}

Der Verfasser beschreibt Cordocythere phreaticola n. g. n. sp. und Eucypris petkovskii n. sp. sowie einige systematische Bemerkungen über 13 Arten, für das erste Mal in der Rumäniens Fauna erwähnt.

Er gibt eine genaue Beschreibung der Mandibel und Maxille von Metacypris cordata, die für die Systematik der Tribus Metacyprini nov. sehr wichtig sind. Die vergleichende Analyse über die Struktur der Antenna I, der Mandibel und Maxille den Gattungen Limnocylhere, Cytheridella, Comphocythere, Metacypris, Elpidium und Cordocythere gaben dem Autor die Gelegenheit die Existenz von zwei phylogenetischen Reihen in der Unterfamilie der Limnocytherinae Sars, 1925 zu erblicken. Sie wurden Tribus Limnocytherini nov. und Tribus Metacyprini nov. genannt.

Die Wiederentdeckung des Isocypris arnoldi für das erste Mal nach seiner ersten Beschreibung ist sehr merkwürdig vom systematischen und zoogeographischen Standpunkt.

Die Anwesenheit der termophilen Arten Dolerocypris sinensis, Stenocypris major und Heterocypris maura im Süden Rumäniens und der phreatophilen Art Cypridopsis subterranea im Nord-Osten des Landes ist interessant für die Zoogeographie.

Durch die aktuelle Verteilung der Representanten des Tribus Metaryprini, ist Cordocythere phreaticola als eine preglaziäle Relikt betrachtet.

\section{TRAVAUX CITES}

ALM (G.). 1914. - Beiträge zur Kenntnis der nördlichen und arktischen Ostracodenfauna. Ark, f. Zool. 9 (5) (Apud Brorstein 1947).

BELdescu (ST.). 1961 a. - Ostracode din complexul de bălţi CrapinaJijila. Com. Acad. R.P.R. 11 (8) : 7-13, 5 fig.

Beldescu (St.) 1961 b. -- Heterocypris rostrata nov. sp. din împrejurimile Brăilei. Com. Acad. R.P.R. 11 (8) : 939-943.

Bhonstein (Z. S.). 1947. - Ostracodes des eaux douces. Faunc de I'U.R.S.S., Crustacés 2 (1) : 1-339, plates 1-14 text figs. 1-206, Moscou-Leningrad (Ed. Acad. Sci. U.R.S.S.).

Cverkov (L.). 1959. — Materiali vrhu slatkovodnata ostracodna fauna v Blgarija. Bull. Inst. Zool. Acad. Sci. Bulgara 8 (Apud Petkovski $1960)$.

D.dDAY (E.). 1900. -- Ostracoda Hungariae. 1-330, Budapest.

Danielopor. (D.) et Vespremeanu (E.). 1964. - The presence of ostracodes on the floating fen soil in Rumania. Fragm. Balcan. Mus. Maced. Sc. Nat. 5 (7) : 29-35, 3 flg.

Dubousky (N. W.). 1927. - Ueber zwei neue Ostracoden im Bassin des Sewerny (Nord) Donetz (Nebenfluss des Don) Zool. Anz. 73 : 81-88, Abd. 1-18. 
Fergusson (E.). 1964. - Stenocyprinae, a new subfamily of freshwater cyprid ostracods (Crustacea), with description of a new species from California. Proc. Biol. Soc. Washington 77 : 17-24, 6 fig.

Gauthier (H.). 1938. - Ostracodes continentaux récoltés par M. Monod au Sahara Occidental et en Mauritanie. Bull. Soc. Sc. Nat. Maroc, $17: 39-50$.

HeRrst (I.). 1951. - Isocypris quadrisetosa Rome (Crustacea, Ostracoda) aus der Eider. Abh. naturw. Ver. Bremen 32 : 403-414 (Apud Triebel. 1959).

JANCARIK (ANT.). 1952. - Herpetocypris chevreuxi (Ostracoda) a new plant parasite of vegetable. Fol. Zool. Entom. 1 (XV) : 111-114.

KLIE (W.). 1935. - Ostracoda aus dem Tropischem Westafrika in Voy. Alluaud et Chappuis en Afrik Occ. Française. Arch. Hydrob. 28 : 35-68, figs. 1-62.

KIIE (W.). 1937. - Ostracoden und Harpacticiden aus brackigen Gewässern an der bulgarischen Küste des Schwarzen Meeres. Mitt. kgl. Naturw. Inst. Sofia, $10: 1-42$.

KLIE (W.). 1938. - Ostracoden aus unterirdischen Gewässern in Süditalien. Zool. Anz. 123 : 148-154.

KLIE (W.). 1938. - Krebstiere oder Crustacea III : Ostracoda, Muschel krebse - in Danl, Die Tierwelt Deutschlands. $34: 1-230$ text figs. 1-786, Jena (G. Fischer).

KIIE (W.). 1943. - Ostracoden aus dem Grundwasser der Umgebung von Kolozsvar. Fragmenta faunistica Hungarica, 6 : 35-41.

LeRuth (R.). 1961. - La biologie du domaine souterrain et la faune cavernicole de la Belgique. Mém. Mus. Roy. Hist. Nat. Belgique. 87.

Löffler (H.). 1961. - Zur Limnologie der Steppenseen. Verh. Inter. Verein. Limnol. 14 : 1136-1141.

LöfFler (H.). 1963. - Die Ostracodenfauna Osterreichs in : Beiträge zur Fauna Austriaca. Silzungsberichte. Mathem-naturw, Kl. Abt. I, 3-5 : 193-211, 1 text abd. und 3 taf.

Lowndes (A. G.). 1932. - Herpetocypris palpiger, a new species of freshwater ostracoda. Ann. Mag. Nat. Hist. 9 : 155-157.

Marazanof (F.). 1965. - Ostracodes de Camargue. Ann. Limnol. 1 (1) : 95-102.

Masi (L.). et Jaquet (M.)). 1906. - Faune de la Roumanie. Ostracodes récoltés par M. JaQuet et déterminés par M. le $\mathrm{D}^{\mathrm{r}}$ L. Masi. Bull. Soc. Sci. de Bucarest 19 (6) : 647-665.

Masi (L.). 1932. - Ostracodi. Escursione Zool. all'Oasi di Marrakesch. Boll. Zool. 3 (5) (Apud KliE 1937).

MülLler (G. W.). 1900. - Deutschlands Süsswasser-Ostracoden. Zoologica 30 (Apud WaGLeR, 1937).

Mülten (G. W.). 1912. — Ostracoda in « Das Tierreich ». Eine Zusammenstellung und Kennzeichnung der rezenten Tierformen. 1-434.

Petkovski (T.). 1957. - Zweiter beitrag zur Kenntnis der Ostrakodenfauna Jugoslaviens. Folia Balc. 1 (9) : 51-56, 8 fig.

Petkovski (T.). 1960. - Süsswasserostracoden aus Jugoslavien VII. Fragm. Balcan. Mus. Maced. Sc. Nat. 3 (12) : 99-106.

Petrovski (T.). 1960. - Zur Kenntnis der Crustaceen des Prespasses. Fragm. Balc. Mus. Maced. Sc. Nat. 3, 15 (74) : 117-131, 135 flg. 
Petkovski (T.). 1964. - Bemerkenswerte entomostraken aus Jugoslavien Acta. Mus. Maced. Sc. Nat. 9, (7) : 143-181.

Plesa (C.), Botea (F.), Racovita (G.). 1964. - Cercetări asupra faunei biotopurilor acvatice subterane din bazinul Crişului Repede. I. Valea Misidului şi afluenţii. Lucr. Inst. Speol. 3 : 367-396.

Pinto (I. D.), Sanguinetti (Y.). 1961. - Observations on Metacypris (Ostracoda) from the Mesozoic of North America and Africa. Esc. Geol. P. Alegre. Bol., 9 : 3-14.

Pinto (I. D.), Sanguinetti (Y. T.). 1962. - A complete revision of the genera Bisulcocypris and Theriosynoecum (Ostracoda) with the world geographical and stratigraphical distribution (including Metacypris, Elpidium, Gomphocythere, Cytheridella). Escol. Geol. P. Alegre Publ. 4 : 1-165.

Ponyi (J.). 1962. - Zoologische Untersuchungen der Röhrichte des Balaton. I. Krebse. Annal. Biol. Tihany, 29 : 129-163.

Pusciriu (V.). 1951. - Contribuţii la cunoaşterea răspîndirii geografice a Ostracodelor de apă dulce din R.P.R. Bul. Stiin. Acad. R.P.R. Sect. St. biol., agron., geolo., geogr. 3 (4) : 667-668, 2 foto, 2 hărți.

Redeke (H. C.)., Den DulK (A.). 1940. - Ostracoda of the Netherlands. Arch. Neerl. Zool. 4 (23) : 139-148.

Rome (D. R.). 1947. - Contribution à l'étude des Ostracodes de Belgique. I. I.es Ostracodes du Parc Saint.-Donat à Louvain. Bull. Mus. Roy. Hist. Nat. Belgique 23 (34) : 1-24, Abd. 1-3.

RoMe (D. R.). 1962. - Ostracodes in « Exploration Hydrobiologique du lac Tanganyka ». Inst. Roy. Sc. Nat. Belgique, 3 (8) : 1-305, 82 tig.

S.IRS (G. O.). 1922-1928. - An account of the Crustaces of Norway. Ostracoda. 9, 1-16 : 1-277, Bergen.

Srrıur. 1952. - Mikropaläontologische Untersuchungen im Tertiär zwischen Ehingen und Ulm a. d. Donau. Geol. Jahrb. 66 : 433-523 (Apud Tressler et Sanguinetti 1962).

Thienemann (A.). 1950. - Die Binnengewässer, Band XVIII Verbreitungsgeschichte der Süsswassertierwelt Europas. 1-809 $+\mathrm{rx}$ pp. Stuttgart.

Tressler (W.). 1941. - Ostracoda from Puerto Rican Bromeliads. $J$. Wash. Ac. Sc. 31 (6) : 263-269.

Tressler (W.). 1956. - Ostracoda from bromeliads in Jamaica and Florida. J. Wash. Ac. Sc. 46 (10) : 333-336, 8 fig.

Tribber (E). 1953. - Genotypus und Schalen-Merkmale der Ostracoden Gattung Stenocypris. Senckenbergiana 34 (1/3) : 5-14.

Triebel (E.). 1959. - Zur Kenntnis der Ostracoden Gattungen Isocypris und Dolerocypris. Senck. Biol. 40 (3/4) : 155-170.

Viehmann (I.), Piesac (C.), Rusu (Th.). 1964. - Peştera dela VaduCrişului. Lucr. Inst. Speol. 3 : 49-82.

Wagler (E.). 1957. -- Crustacea. Die Tierwelt Mitteleuropas II, 2 a : 19-70.

Wol.F (J. P.). 1919. - Die Ostracoden der Umgebung von Basel. Arch. f. Naturgesch. Abt. A, $85: 1-80$.

(Institut de Spéologie «E. Racovitza», Bucarest, Roumanie). 